\title{
Article \\ An Empirical Investigation of GIS Interoperability Best Practices In Industry
}

\author{
Allan Mazimwe ${ }^{1, *}\left(\mathbb{D}\right.$, Imed Hammouda ${ }^{2,3}$ and Anthony Gidudu ${ }^{4, *}$ \\ Makerere University, Uganda; allanmazimwe@cedat.mak.ac.ug \\ Chalmers and University of Gothenburg, Sweden; hammouda@chalmers.se, \\ 3 Mediterranean Institute of Technology, South Mediterranean University, Tunisia; \\ imed.hammouda@medtech.tn \\ 4 Makerere University, Uganda; agidudu@cedat.mak.ac.ug \\ * Correspondence: allanmazimwe@cedat.mak.ac.ug; Tel. +256 774322196
}

\begin{abstract}
Reuse of patterns is a self-evident approach for managing interoperability concerns. Although patterns for resolving interoperability barriers exist in the literature, no study exists on adoption of interoperability patterns by Geographic Information Systems (GIS) practitioners in industry. Thus there is limited understanding of pattern re-usability, yet the advantages offered by interoperability patterns provide a reasonably sound justification for their usage. This paper examines the adoption of proven interoperability best practices in the GIS industry. An empirical study that involved the use of semi-structured interviews was employed to gather data from GIS developers on domain interoperability best practices. Results indicated that industry and communities of practice have been converging on the technical level to ensure interoperability of GIS concerns. Semantic interoperability and related patterns are least understood, yet semantic barriers still exist. This is partly due to the complexity associated with the top-down approach used to develop semantic interoperability solutions. Therefore, this study proposes research into resolving barriers in the adoption of interoperability patterns that reduce complexity while solving semantic interoperability barriers.
\end{abstract}

Keywords: Re-usability; Patterns; Interoperability; Geographic Information Systems

\section{Introduction}

Interoperability is considered a critical success factor for ensuring public service delivery in various domains such as the health, environment and disaster management domains. A combination of interoperability concepts and proven shared best practices are required to overcome barriers in sharing of resources between multiple systems[1]. However, implementing interoperable solutions requires an adequate understanding of the theory and methods underpinning the interoperability barriers involved. For instance, to implement a semantic interoperable GIS, a domain expert has to understand the complex notions associated with ontology development which requires the support of a knowledge engineer[2]. Patterns in GIS have been proposed as proven best practices for documenting good design practices for reuse, ideally enabling inexperienced domain experts to construct high-quality interoperable solutions[3] without much help.

Although interoperability pattern use exists in literature, there is still limited understanding about pattern adoption for solving interoperability barriers in GIS industry. Knowledge of pattern adoption for managing interoperability concerns is necessary to trigger off research in overcoming re-use barriers associated with interoperability patterns in industry. Reuse of these patterns ensures resolution of re-occurring interoperability problems with existing and well-tested solutions which shortens the development time and cost, whatever the task[3]. Pattern reuse also provides a fallback level that ensures minimal interoperability while still maintaining heterogeneity[4]. The promises offered by interoperability patterns in managing interoperability concerns provide a reasonably sound justification for their usage by industry. 
Therefore, the following research question was adopted; "what existing and emerging best practices are useful to domain experts while managing interoperability concerns in the GIS industry?" To answer the research question, a qualitative study was used to provide an insight into interoperability best practices in the industry since no such study existed. The contribution of this paper is that it provides an understanding of GIS interoperability requirements/scenarios and emerging best practices for managing these requirements. The paper also discusses implications for interoperability pattern research in GIScience and industry. This paper is structured as follows: The background and related work are presented in section 2, data collection and analysis procedure in section 3, results and discussions in section 4 and 5 respectively. Section 6 contains the conclusions and future work.

\section{Background and Related Work}

This section presents related work on general interoperability concepts (Section 2.1) and, interoperability patterns (Section 2.2).

\subsection{Interoperability}

Chen[5] defines interoperability as "the ability to communicate and exchange information, use the information exchanged and provide access to the functionality of a third system". Different types of interoperability barriers exist in GIScience depending on the interoperability framework being used i.e Technical, semantic and organizational barriers[6]. Technical interoperability barriers are associated with the inability of different systems (e.g Web Services/application programming interfaces (APIs)) to operate together to reach a common task or goal. With technical interoperability, data does not necessarily interoperate unless variability in syntax and semantics is eliminated. To a larger extent, there is some agreement that syntactic interoperability in a GIS can be achieved through rigid standardization such as standards from Open GIS Consortium(OGC) and International Standardization Organization Technical Committee 211(ISO TC211) [4,7].

Even with robust standardization, semantic interoperability barriers continue to exist as standard driven products and efforts are often developed in isolation thus reflecting different conceptualizations and semantic drifts when combined[3]. Semantic interoperability barriers cannot be resolved through standardization since they rely on meanings of concepts in data remaining invariant during the exchange between multiple systems. According to Kubicek and Cimander[7], theoretically proven methods that constrain meaning using ontologies have been developed, but with some practical implementation problems. The last barrier is the organizational interoperability which still has vague concepts and lacks conceptual clarity as it usually boils down to the willingness of organizations / data owners to share Geoinformation. Together with interoperability concepts, patterns have been proposed as a way of overcoming the interoperability barriers discussed[1,3,8].

\subsection{Interoperability Patterns}

Patterns science provides a means of solving re-occurring problems in a particular context using proven solutions [9]. For instance, the Mediating Connector Patterns[10] have been used to manage interoperability in Information systems. Danko [11] defines different enablers for interoperability in GIScience. These include shared proven best practices and interoperability concepts such as authorization, copyright, standards, business models, infrastructures, metadata, support for multiple languages, coordinate reference systems, views among others. In essence interoperability patterns ${ }^{1}$ are best practices and therefore interoperability enablers since they provide proven solutions to re-occurring interoperability problems in a particular context.

1 All patterns are best practices but the converse is not true. 
In the technical interoperability ecosystem, explicit work using interoperability patterns to manage concerns has been explored in the Internet of Things (IoT)[12], enterprise information systems ${ }^{2}$ and health information systems[8]. Additionally, organizational interoperability patterns have been identified in the health domain $[1,8]$ but can validly be applied in any other domain. In the semantic interoperability ecosystem, ontology design patterns $\left(\mathrm{ODPs}^{3}\right)$ have been used as common strategies and building blocks that act as an interoperability fall back level $[3,13]$ while at the same time supporting heterogeneity for participating data. Krisnadi et al [14] illustrate a concrete use-case where ontology design patterns in a federated architecture are used to manage semantic GIS data integration for heterogeneous repositories. The work of Krisnadi[15] provides proof of concept that patterns can be used to manage GIS interoperability concerns(i.e. spatial data, Geo-services and Geoprocesses).

Different inductive approaches to pattern development have been proposed each of which has its own drawbacks. These include; development workshop, enhancement workshop, guided development, shepherding, expert interview, observation, open channel, literature review, collaborative learning and development, pattern mapping and pattern writing [16]. To make it easier for domain experts to select the right pattern to apply for a given interoperability challenge, different pattern classifications schemes/criteria exist. Patterns can be classified by; discipline[17], domain (see classification by similar usage[18]), paradigm, granularity[19], scope etc. Since interoperability occurs at different levels of granularity in an information system, this study uses classification by granularity to provide insight on related work in the GIS interoperability ecosystem.

\subsection{Pattern Classification}

Patterns can be classified as at coarse scales as application domain patterns, architectural patterns; and at finer scales as design patterns, syntactic patterns and semantic patterns[18]. Arguably, these patterns can be thought as architectural patterns at various granularities i.e. application, system, macro and micro architectural levels. While this classification indicates the separation of the levels as a difference in granularity, some patterns can exist at different levels.

\subsubsection{Domain Patterns}

These concern the purpose or overall usage of an object in a domain. May not be useful in a specific construction context instead they are concerned with activities before or actual construction examples include knowledge patterns, abstract architectural patterns.

\subsubsection{Architectural Patterns}

These patterns describe the overall coarse structuring of objects in question whether its $a$ software, an ontology or building etc, how to divide them into subsystems, the responsibilities of the subsystems and their relations. These patterns can be grouped as software architectural patterns, Knowledge architectural patterns, data model patterns etc. Example of interoperability patterns used in GIS includes Service-oriented Architecture (SOA) patterns, exchange patterns such as service Choreography-orchestration [20], federated pattern as the one used by Krisnadi[15] for semantic-based spatial data integration of heterogeneous repositories, publish-find-bind pattern [21].

\subsubsection{Design Patterns}

At detailed and micro levels, there exists a set of patterns that describe ways to design the architectural components and their interactions, but they are still independent of representation language. These patterns can be an analysis patterns, software design patterns (e.g. abstract factory

\footnotetext{
See catalog http://project-interoperability.github.io/exchange-patterns/ See catalog: http:// ontologydesignpatterns.org/wiki/Community:ListPatterns
} 
pattern [22]), data model patterns (selected parts) or a small named collection of semantic patterns such as ODPs that have been suggested as re-usable solutions for semantic interoperability barriers[13,23].

\subsubsection{Semantic Patterns}

At a finer detail level, there exists semantic Patterns that commonly represent a concept but independent of the representation language. The meaning of a certain idiom belonging to the Syntactic Pattern level could in turn constitute a Semantic Pattern. Examples include Meta-modeling languages, modeling languages e.g. UML, semantic patterns for ontologies [23], Knowledge patterns.

\section{Research Methodology}

The methodology is divided into two parts i.e data collection procedure and data analysis. In this section, we address three research questions:

- RQ1: What GIS interoperability scenarios/requirements exist?

- RQ2: How useful are interoperability patterns in managing GIS concerns?

- RQ3: Are there any other new emerging interoperability best practices?

\subsection{Data Collection Procedure}

To identify interoperability scenarios and corresponding best practices, case study assessments were undertaken on eight data points representing multinational GIS industry organizations. Each of these organisations has multiple GIS software packages and projects in different countries. Snowball sampling was done to identify respondents from these organisation to provide a global view of the different projects and products i.e skilled GIS developers with 10 and more years of experience. An empirical study that involved the use of semi-structured interviews was employed to gather data from respondents on interoperability practices based on different projects and products delivered within the organisation. For confidentiality purposes, aliases R1, R2, R3, R4, R5, R6, R7, and R8 are used to denote the responses.

Respondent R1 (based in Italy) provides open GIS solutions for land registration and mapping implemented in over 5 countries globally. R2 (based in the USA) is an international supplier of GIS software, geodatabase management and webGIS applications such as Software As A Service (SAAS) mapping platform, GIS server applications, focused GIS apps, GIS data with over 80 distributors on all continents. R3 represents a French company that implements over 40 international projects in about 30 countries on data infrastructure, development of geoportals and land information systems among others. R4 represents an Israeli based company that provides enterprise GIS solutions integrated with enterprise resource planning systems(ERP) capabilities, 3D GIS for utility mapping, and physical planning applications in over 20 countries.

R5 represents a Canadian firm that provides GIS consulting services in agriculture, location-based services, and environmental modeling. R6 represents an in-house GIS integration effort in a public authority in Uganda. R7 represents a Canadian based GIS company implementing Geoinformation management solutions that publish, integrate, and enable access between enterprises over the cloud. R8 represents a German/Swiss company that provides integration of GIS resources for utility, industrial plant management, transport and logistics markets applications with enterprise resources.

\subsection{Data Analysis}

An inductive approach towards interview analysis was used to explore interoperability requirements/scenarios and best practices for managing interoperability in the industry. The interview data was transcribed in ATLAS.ti. The resulting unstructured data of interview was reconstructed into sentences that were subsequently coded. The codes were then grouped according to themes in the data. On the other hand, the structured part of the interview data, the responses are coded as very useful (+), not sure/not applicable (0) and not useful (-). Once the entirety of the material was coded, the 
researchers reviewed the examined theme ideas of the interview material and how they relate with each other as regards to GIS interoperability best practices(both patterns and other practices).

\section{Results}

This section presents results with respect to the research questions in section 3.

\subsection{What interoperability scenarios/requirements exist?}

Figure 1 shows the nature GIS systems, evolution and thus the need for interoperability in industry. The requirement for interoperability and system accessibility increases from desktop to cloud GIS applications as indicated in Figure 1. The choice of technology is dependent on requirements and nature of the client.

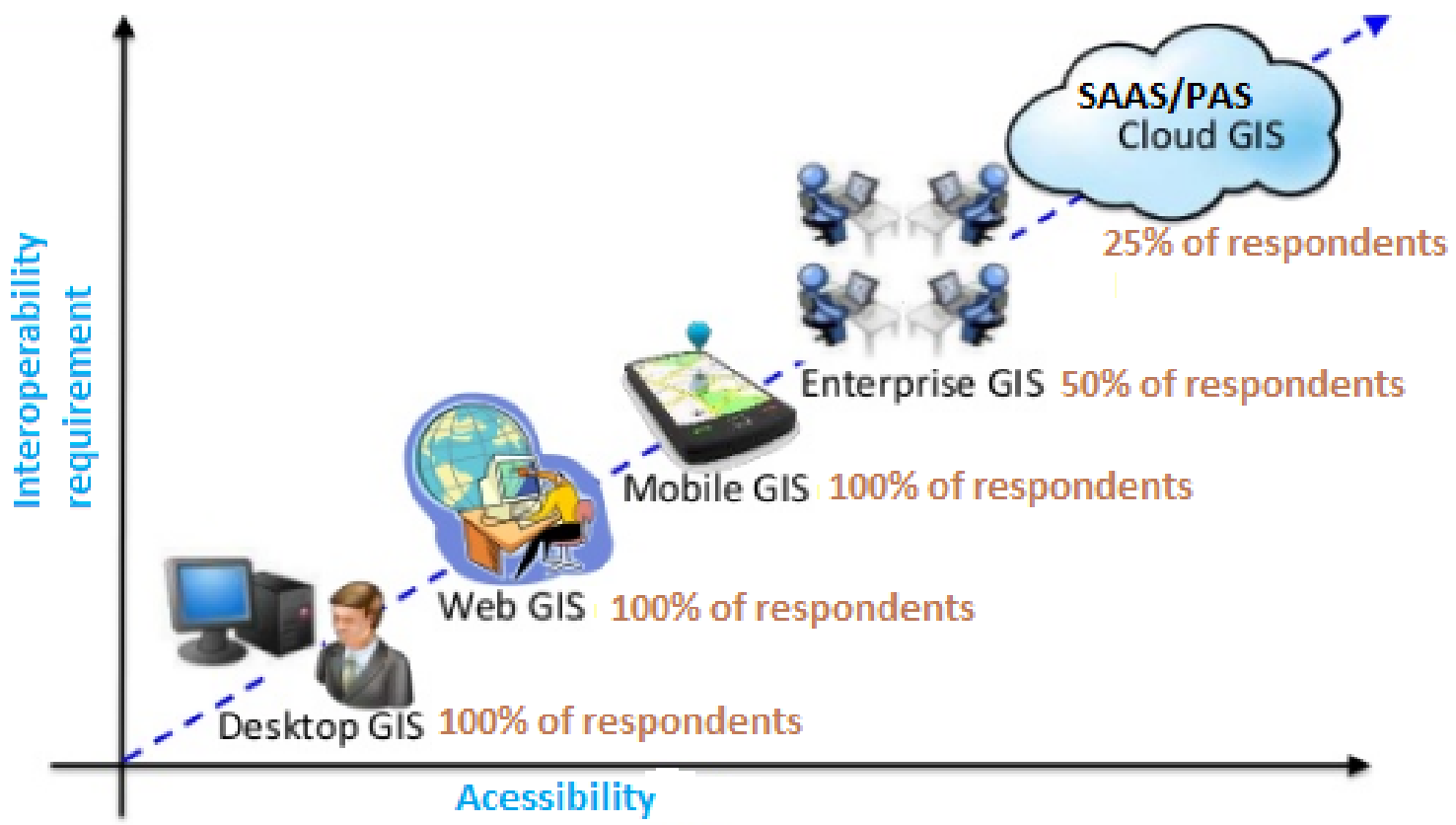

Figure 1. GI Systems in industry

Respondents indicated that all the systems are expected to interoperate with either internal or external systems e.g bank systems, billing systems, Land and Property Systems (LAPs) etc. These systems share concerns ${ }^{4}$ in either a one-way or two-way direction in such a manner that only access to required geoinformation is granted while manipulation and management are left to the parent interoperating systems.

Table 1 presents existing, desirable aspects and reasons for GIS interoperability. Results indicate that respondents mostly use standards, standardized web services, direct data access and common data model constructs to leverage interoperability. $37.5 \%$ of the respondents use bottom-up APIs which are driven by business assets rather than client requirements. In addition, interoperability APIs, Service-API/ service-database integration, security and mechanisms to guarantee availability are most desirable requirements of an interoperating GIS. These scenarios and requirements indicate that there is an adoption of mechanisms in the ecosystem for managing technical interoperability barriers. On the other hand, semantic driven scenarios exist but were less understood and with lower adoption. This is possibly due to two reasons. Firstly, the roles of the domain expert and ontology engineer are separate which slows down the ontology development process in the individual case by requiring additional

4 i.e spatial data/information, services, and processes) 
Table 1. Existing and desired interoperability scenarios.

\begin{tabular}{|c|c|}
\hline Interoperability scenario & Respondents \\
\hline Existing scenario & $37.5 \%$ \\
1- Bottom-up driven interoperability API & $0 \%$ \\
3- Direct data access and integration & $62.5 \%$ \\
4- Using standards e.g OGC/ISO TC211 & $100 \%$ \\
5- Using common data model constructs & $75 \%$ \\
6- Using standardized web services e.g OGC WMS & $100 \%$ \\
7- Use of ontology and semantic technologies & $37.5 \%$ \\
\hline Desired scenario and requirements & $50 \%$ \\
2- Service to service/API integration & $37.5 \%$ \\
3- Service to database integration where the target system & $37.5 \%$ \\
does not provide suitable services or interoperability API. & $100 \%$ \\
4- Security and mechanisms to guarantee service and data availability & $12.5 \%$ \\
5- Need to ensure data validity and integrity of interoperated data & $12.5 \%$ \\
6 - Existence of documented interoperability best practices & \\
\hline Why interoperability? & $100 \%$ \\
\hline 1- To allow the functionality of GIS Software to be extended to support \\
user requirements that are complementary to the application domain. \\
2- To integrate and / or consume data/services/functionality \\
of complimentary systems. \\
3- Need for improved efficiency \\
\hline
\end{tabular}

tasks to be performed. Secondly, the top-down approach to ontology development requires a steep learning curve for practitioners in the domain due to abstract notions associated with ontologies. Bridging these gaps with patterns / proven best practices (as indicated in Table 1) could go a long way towards furthering the adoption of semantic technologies among non-academics and domain experts.

Figure 1 shows a high adoption of GIS on the web which presents an opportunity for GIS to leverage Geospatial semantic web research and technologies for interoperability. Finally, results indicate the need to ensure data validity and integrity of interoperated data.

\subsection{How useful are interoperability patterns in managing GIS interoperability Concerns?}

This section provides a list of patterns preferred for leveraging interoperability for Geographic information systems as evaluated by respondents (see Table 2). The responses are coded as very useful $(+)$, not sure/not applicable (0) and not useful (-). In this paper, pattern usefulness is used as an indicator of pattern adoption in industry. Table 2 categorizes best practices as architectural, semantic, and data model patterns. In Table 2, respondents were familiar with patterns at coarse more than those at finer levels of abstraction. For instance, architectural patterns that provide generic structuring of components in GISystems in the context of technical interoperability appear most common in Table 2.

The n-tier architectural pattern (2tier, 3tier), query/response pattern, SOA patterns, workflow patterns and model view controller patterns were found to be the most useful for designing interoperable solutions. The choice of the patterns selected while designing interoperability solutions is driven by opportunistic and pragmatic requirements such as existing functional and quality requirements, design constraints rather than strategic thinking. However, respondents least understood finer patterns which compose the architectural pattern components. For instance, software design patterns, ontology design patterns are compositions of architectural components were least highlighted. Worthy to note is that none was aware of any ontology design patterns. Thus the results in Table1 and Table 2 confirm the notion that many industry sectors and communities of practice have been converging on the technical level to ensure interoperability of GIS concerns (such as data, services etc).

Technical patterns such as architectural patterns and software design patterns only ensure technical interoperability. However, for data-intensive systems, semantic heterogeneity issues could 
Table 2. Pattern usefulness.

\begin{tabular}{|c|c|c|c|c|c|c|c|c|c|}
\hline Pattern Name & R1 & R2 & R3 & $\mathrm{R} 4$ & R5 & R6 & R7 & R8 & useful (\%) \\
\hline \multicolumn{10}{|l|}{ Architectural patterns } \\
\hline n-tier server architecture & + & + & + & + & + & + & 0 & + & 87.5 \\
\hline Cross platform access & 0 & + & + & - & + & - & + & + & 62.5 \\
\hline Platform independence & 0 & + & + & - & + & - & - & - & 37.5 \\
\hline Cross application domain access & 0 & + & + & - & - & - & + & + & 50 \\
\hline Platform scale independence & 0 & + & + & - & - & - & + & + & 50 \\
\hline Higher level service facade pattern & 0 & 0 & + & + & - & + & + & - & 50 \\
\hline Model View controller pattern & - & + & + & - & - & - & + & - & 37.5 \\
\hline SOA patterns-Rest API & + & + & + & + & + & + & + & + & 100 \\
\hline Data model transformation patterns & 0 & 0 & 0 & + & - & 0 & - & - & 12.5 \\
\hline Query/Response pattern & + & + & + & + & + & + & + & + & 100 \\
\hline Federated query pattern & - & + & + & + & - & - & + & + & 62.5 \\
\hline Identities pattern & + & + & + & + & - & + & + & + & 87.5 \\
\hline Federated identities pattern & + & + & + & - & - & - & + & + & 62.5 \\
\hline $\begin{array}{c}\text { Coordination pattern(s) } \\
\text { (Orchestration, Choreography) }\end{array}$ & - & 0 & + & - & - & - & + & + & 37.5 \\
\hline Workflow pattern & + & + & + & + & - & + & + & + & 87.5 \\
\hline Broadcast pattern & - & - & + & + & - & + & + & + & 62.5 \\
\hline Publish- Find-Bind pattern & - & + & - & - & - & - & + & - & 25 \\
\hline GeoKnowledge design pattern & - & + & - & + & - & - & - & - & 25 \\
\hline \multicolumn{10}{|l|}{ Other identified architectural patterns } \\
\hline Gateway integration pattern & + & 0 & 0 & 0 & 0 & 0 & 0 & 0 & 12.5 \\
\hline \multicolumn{10}{|l|}{ Data model patterns } \\
\hline Data model patterns-UML convention & + & + & + & + & - & + & + & - & 75 \\
\hline \multicolumn{10}{|l|}{ Semantic design patterns } \\
\hline Ontology design patterns & - & - & - & - & - & - & - & - & 0 \\
\hline
\end{tabular}

still arise. For instance, independently developed and maintained data sources could have a heterogeneous schema. The differences may lie in the use of distinct vocabularies, different levels of granularity in modeling the data, or conflicting conceptualization rather than the ability of software component to interoperate. This will present a semantic interoperability challenge which can neither be solved by common standards but rather by constraining meaning via ontologies. While many ontology design patterns have been presented and used in various system development projects as agents for improving semantic interoperability[13], GIS industry has not fully adopted these patterns as indicated in Table 2.

\subsection{Are there emerging interoperability best practices?}

Results also indicate that there exist anti-patterns for data integration and discovery in industry. For instance, $37.5 \%$ of respondents recommended the use of an aggregated view to integrate data and search over it. In this case, data tables are joined together and queried. Aggregated views are efficient for small data sets in a single repository but may prove inefficient in federated systems that answer queries on cross-repository datasets. In such instances, an aggregated view pattern could be a common practice but rather ineffective and risks being highly counterproductive with multi-repository data integration and discovery. Respondents further identified the need for patterns and corresponding pattern documentation to support novice users in pattern discovery, understanding and use for different interoperability solutions. Pattern re-use together with exemplary use-case scenarios is key to pattern adoption in the GIS ecosystem. Exemplary use-case scenarios will act as a guideline for novice users.

From a process point of view, another emerging best practice indicated was the need to focus on agile processes which collaborate, self-organizing stakeholders involved in implementing interoperability solutions. Agile processes will typically involve domain experts (GIS experts), 
developers and managers etc. to holistically focus on necessary aspects of interoperability. From a tooling point of view, respondents indicate the need for tooling improvements to support a well-developed agile methodology with patterns. A modular approach towards data integration and discovery solution developments that don't require experts to know detail was also suggested as an area for development.

\section{Discussion}

This section presents a discussion of results and possible research directions to enable pattern adoption for solving interoperability challenges. Furthermore, the implication of study to industry and threats to validity are also discussed.

\subsection{Implications for Research}

Results indicate limited adoption of patterns for solving GIS semantic interoperability barriers. This is possibly because ontology engineers are still most commonly academics and researchers, and the industrial uptake of semantic technologies is not as high as it could be as pointed out by Hammar[24]. One powerful driver for pattern adoption in the GIS community is the existence of well-documented catalogs for patterns required in solving interoperability challenges. There exists a few ODPs and technical pattern catalogs developed and specialized for interoperability in the geospatial community. Therefore, there is a need to identify more such patterns which when coupled with good exemplary uses-cases for managing interoperability concerns such as that in [15] will be important drivers for pattern adoption. Such other examples could include Ontology design pattern driven web service composition in GIS amongst others. Presutti et al [25] have developed an agile driven extreme design (XD) methodology and subsequent tooling. While Hammar[26] provides an improvement of the tooling support for agile XD for novice users, there is still room for more research. For example, pattern recommendation to a novice user can be enhanced to capture pattern language concepts discussed in Hitzler et al [27], where common patterns can be used to search other related patterns. Last but not least, domain experts in the industry are interested in simple, understandable and quality patterns that can be used to solve the requirements at hand. Important to note is that a quality model for ODPs has been developed already[24], however, more research can be done to ensure that industry practitioners can decide for themselves which quality characteristics to prioritize for data/service interoperability solution developments.

\subsection{Implications for Industry}

It is important that domain experts/developers play a leading role in interoperability solution engineering. The ease associated with identifying quality interoperability patterns offers a possibility for re-use of existing proven best practices. For instance, data integration leveraging semantic technologies can be made easier and understandable through simple to use ontology design patterns thus making it possible for GIS developers in the industry to re-use them. On the other hand, agile methodologies and tooling improvements for pattern-based engineering ensures that novice GIS users can co-develop easy to modify interoperability solutions for deployment in different contexts without much help from specialized experts (e.g knowledge engineers and mainstream IT developers). The study also indicates that anti-patterns are used by practitioners for interoperability solution development. Research into identifying and documenting such bad interoperability practices that are otherwise thought to be good, could be worthwhile. This will enable practitioners to avoid selection and reuse of counterproductive practices.

\subsection{Threats to validity}

Internal Validity: The approach of pattern identification by interviewing domain experts will result in patterns that are dependent on the expertise of respondents and relevance or future pattern appliers. Other relevant patterns can be identified using methods mentioned in Günther and Knote [16]while 
considering their constraints. Our research is however interested in the industry which gives us a picture of interoperability best practices in the domain.

External Validity/generalisability: The target population for this research GIS solution designers, developers, and maintainers who had some form of experience with data integration (10 years and more). The respondent firms offer different applications and have a presence in over 100 countries in different application domains like land administration, GIS data integration, environmental modeling navigation amongst others which would render our results generalizable. The respondents in some way provide a global market reflection for different software products in the firms they work.

Construct Validity: To overcome threats due to the valid interpretation of questions, we clarified the meanings of terms to respondents and provided a background to the interview questions. In a combination, open-ended questions were used to allow the participant to elaborate on answers. All data were collected with a recording device (i.e. voice) and by writing to avoid researcher bias. To achieve reliability, different researchers with different expertise (GIS and software engineering experts) assisted in data collection.

\section{Conclusions}

Patterns play a crucial role in managing interoperability barriers and concerns in GIScience. In this study, we investigate interoperability scenarios/requirements in industry and usefulness of proven interoperability best practices for managing these requirements in the GIS. From the study, patterns have been used to achieve technical interoperability in industry. However, not much evidence exists to suggest that semantic technologies and associated patterns are often used by industry practitioners to solve interoperability barriers. Best practices such as agile methodologies with adequate tooling support were also identified for building interoperable GIS solutions. While patterns provide re-usable solutions to solve interoperability barriers, there also exists anti-patterns which seemingly are good practices but produce counterproductive results in some scenarios. This paper also presents a discussion of possible research directions. Some of these research directions include pattern identification and documentation, tooling improvements for agile based methodologies and pattern concepts used in interoperability solution development. This research agenda together with more use cases where patterns have been used to manage interoperability concerns provides a lot of promises for pattern adoption and re-use in GIS industry.

\section{References}

1. Milosevic, Z. Addressing interoperability in E-health: an Australian Approach. 10th IEEE international Enterprise Distributed Object Computing Conference Workshops (EDOCW'06'), 2006.

2. Dimitrova, V.; Denaux, R.; Hart, G.; Dolbear, C.; Holt, I.; Cohn, A.G. Involving domain experts in authoring OWL ontologies. International Semantic Web Conference. Springer, 2008, pp. 1-16.

3. Fritzsche, D.; Grüninger, M.; Baclawski, K.; Bennett, M.; Berg-Cross, G.; Schneider, T.; Sriram, R.; Underwood, M.; Westerinen, A. Ontology Summit 2016 Communique: Ontologies within semantic interoperability ecosystems. Applied Ontology 2017, 12, 91-111.

4. Janowicz, K. Modeling Ontology Design Patterns with Domain Experts-A View From the Trenches. In: Hitzler, P., Gangemi, A., Janowicz, K., Krisnadhi, A., Presutti, V. (eds.) Ontology Engineering with Ontology Design Patterns - Foundations and Applications, Studies on the Semantic Web. IOS Press, 2016, Vol. 25, pp. 233-243.

5. Chen, D. Framework for Enterprise Interoperability, in Enterprise Interoperability. INTEROP-PGSO Vision (eds B. Archimède and B. Vallespir); John Wiley and Sons, Inc.: Hoboken, NJ, USA. doi: 10.1002/9781119407928.ch1, 2017.

6. Manso, M.Á.; Wachowicz, M. GIS design: A review of current issues in interoperability. Geography Compass 2009, 3, 1105-1124.

7. Kubicek, H.; Cimander, R. Three dimensions of organizational interoperability. European Journal of ePractice 2009, 6, 1-12.

8. Authority, N.E.H.T. Interoperability Framework V2, 2007. Accessed: 2018-07-24. 
9. Gamma, E.; Helm, R.; Johnson, R.E.; Vlissides, J. Design Patterns: Elements of Reusable Object-Oriented Software.; Addison-Wesley, Reading, 1995.

10. Spalazzese, R.; Inverardi, P. Mediating connector patterns for components interoperability. European Conference on Software Architecture. Springer, 2010, pp. 335-343.

11. Danko, D.M. Geospatial metadata. In Springer handbook of geographic information; Springer, 2011; pp. 191-244.

12. Bröring, A.; Schmid, S.; Schindhelm, C.K.; Khelil, A.; Kabisch, S.; Kramer, D.; Le Phuoc, D.; Mitic, J.; Anicic, D.; Teniente López, E. Enabling IoT ecosystems through platform interoperability. IEEE software 2017, 34, 54-61.

13. Blomqvist, E.; Hitzler, P.; Janowicz, K.; Krisnadhi, A.; Narock, T.; Solanki, M. Considerations regarding Ontology Design Patterns. Semantic Web 2016, 7, 1-7.

14. Krisnadhi, A.A.; Hu, Y.; Janowicz, K.; Hitzler, P.; Arko, R.A.; Carbotte, S.; Chandler, C.; Cheatham, M.; Fils, D.; Finin, T.; others. The GeoLink Framework for Pattern-based Linked Data Integration. International Semantic Web Conference (Posters \& Demos), 2015.

15. Krisnadhi, A.; Hu, Y.; Janowicz, K.; Hitzler, P.; Arko, R.; Carbotte, S.; Chandler, C.; Cheatham, M.; Fils, D.; Finin, T.; others. The GeoLink modular oceanography ontology. International Semantic Web Conference. Springer, 2015, pp. 301-309.

16. Günther, A.; Knote, R. How to Design Patterns in IS Research-A State-of-the-Art Analysis 2017.

17. Alexander, C.; Ishikawa, S.; Silverstein, M. A Pattern Language: Towns, Buildings, Construction; Oxford University Press, 1977.

18. Blomqvist, E. State of the Art: Patterns in Ontology Engineering; Ingenjörshögskolan, 2004.

19. Meunier, B.F.R.; Rohnert, H.; Sommerlad, P.; Stal, M.; Buschmann, F. Pattern Oriented Software Architecture: A System of Patterns 1996. 1.

20. Hobona, G.; Fairbairn, D.; Hiden, H.; James, P. Orchestration of grid-enabled geospatial web services in geoscientific workflows. IEEE Transactions on Automation Science and Engineering 2010, 7, 407-411.

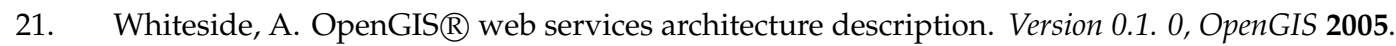

22. Díaz, L.; Schade, S. GEOSS service factory: Assisted publication of geospatial content. Advancing Geoinformation Science for a Changing World 2011, pp. 423-442.

23. Hammar, K.; Lin, F.; Tarasov, V. Information reuse and interoperability with ontology patterns and linked data. International Conference on Business Information Systems. Springer, 2010, pp. 168-179.

24. Hammar, K. Towards an ontology design pattern quality model. PhD thesis, Linköping University Electronic Press, 2013.

25. Presutti, V.; Daga, E.; Gangemi, A.; Blomqvist, E. eXtreme design with content ontology design patterns. Proc. Workshop on Ontology Patterns, 2009.

26. Hammar, K. Ontology design patterns in webprotégé. 14th International Semantic Web Conference (ISWC-2015). CEUR Workshop Proceedings, 2015.

27. Hitzler, P.; Gangemi, A.; Janowicz, K.; Krisnadhi, A.A.; Presutti, V. Towards a simple but useful ontology design pattern representation language. Proceedings of the 8th Workshop on Ontology Design and Patterns (WOP 2017), CEUR Workshop Proceedings, 2017, Vol. 2043.

Author Contributions: In this research all authors contributed equally. All Authors participated in the Conceptualization, Methodology, and Data collection. Data analysis and Writing-Original Draft Preparation were done by Allan Mazimwe; while Editing and supervision by imed Hammouda \& Anthony Gidudu.

Funding: This research was funded by Styrelsen för Internationellt Utvecklingssamarbete (SIDA) grant number BRIGHT317.

Conflicts of Interest: The founding sponsors had no role in the design of the study; in the collection, analyses, or interpretation of data; in the writing of the manuscript, and in the decision to publish the results".

\section{Abbreviations}

The following abbreviations are used in this manuscript: 
11 of 11

$\begin{array}{ll}\text { APIs } & \text { Application programming interfaces } \\ \text { ODPs } & \text { Ontology Design Patterns } \\ \text { GIS } & \text { Geographic Information systems } \\ \text { OGC } & \text { Open GIS consortium } \\ \text { ISOTC211 } & \text { International Standardization Organization Technical Committee 211 } \\ \text { 3D } & \text { Three Dimensions } \\ \text { XD } & \text { eXtreme Design }\end{array}$

